\title{
Methodology for Combined TMS and EEG
}

\author{
Risto J. Ilmoniemi • Dubravko Kičić
}

Received: 6 November 2009/ Accepted: 21 November 2009/Published online: 10 December 2009

(C) The Author(s) 2009. This article is published with open access at Springerlink.com

\begin{abstract}
The combination of transcranial magnetic stimulation (TMS) with simultaneous electroencephalography (EEG) provides us the possibility to non-invasively probe the brain's excitability, time-resolved connectivity and instantaneous state. Early attempts to combine TMS and EEG suffered from the huge electromagnetic artifacts seen in EEG as a result of the electric field induced by the stimulus pulses. To deal with this problem, TMS-compatible EEG systems have been developed. However, even with amplifiers that are either immune to or recover quickly from the pulse, great challenges remain. Artifacts may arise from the movement of electrodes, from muscles activated by the pulse, from eye movements, from electrode polarization, or from brain responses evoked by the coil click. With careful precautions, many of these problems can be avoided. The remaining artifacts can be usually reduced by filtering, but control experiments are often needed to make sure that the measured signals actually originate in the brain. Several studies have shown the power of TMS-EEG by giving us valuable information about the excitability or connectivity of the brain.
\end{abstract}

This is one of several papers published together in Brain Topography on the "Special Topic: TMS and EEG".

R. J. Ilmoniemi $(\bowtie) \cdot$ D. Kičić

Department of Biomedical Engineering and Computational

Science (BECS), Helsinki University of Technology, P.O. Box

2200, 02015 TKK, Finland

e-mail: risto.ilmoniemi@tkk.fi

R. J. Ilmoniemi · D. Kičić

BioMag Laboratory, HUSLAB, Helsinki University Central

Hospital, HUS, Finland

D. Kičić

Viševica Komp d.o.o., Jordanovac 47, 10000 Zagreb, Croatia
Keywords Transcranial magnetic stimulation . Electroencephalography · Cortical excitability · Brain connectivity $\cdot$ TMS $\cdot$ EEG

\section{Introduction}

The combination of transcranial magnetic stimulation (TMS; Barker et al. 1985) with simultaneous electroencephalography (EEG) provides us the possibility to noninvasively probe the brain's excitability, time-resolved connectivity, and instantaneous state. The electric currents induced in the brain by TMS can depolarize cell membranes so that voltage-sensitive ion channels are opened and action potentials are initiated. Subsequent synaptic activations are directly reflected in the EEG (Ilmoniemi et al. 1997), which records a linear projection of the postsynaptic current distribution on the lead fields of its measurement channels (Ilmoniemi 2009). If the conductivity structure of the head is taken into account, the EEG signals can be used to locate and quantify these synaptic current distributions and to make inferences on local excitability and area-to-area functional connectivity in the nervous system (Komssi et al. 2002, 2004, 2007; Massimini et al. 2005).

In the first TMS-evoked EEG recordings by Cracco et al. (1989), transcallosal responses were reported with an onset latency of 8.8-12.2 ms from the pulse. As one could expect, the induced electric field produced large stimulus artifacts in the EEG leads. The same group (Amassian et al. 1992) stimulated the cerebellum and recorded responses from the interaural line. The artifacts were reduced by suitably adjusting the geometrical arrangement between the coil and the electrodes and a scalp-grounded metal strip between them. More advanced methods to deal with the 
electromagnetic interference have been devised, but other artifacts still pose a great challenge to TMS-EEG studies.

Using electronics that decouples the electrodes from the amplifier stages during the pulse, Ilmoniemi et al. (1997) mapped the scalp distribution of the electric potential due to the stimulation of the motor and visual cortices. TMS gave rise to an immediate strong response under the figureof-eight coil. Within 5-10 ms, the activation shifted ipsilaterally; within $20 \mathrm{~ms}$, the contralateral homologous areas were activated. The multiple-channel recording gave the possibility to determine the loci of the evoked neuronal activity, using dipole modeling (Scherg 1992) or minimum-norm estimation (Hämäläinen and Ilmoniemi 1994).

To obtain a good signal-to-noise ratio in EEG measurements, a number of requirements must be satisfied. The recording system must have a low noise level; at the same time, it must be insensitive to or it must recover quickly from the powerful TMS pulse. Magnetic stimulation is usually repeated dozens of times to increase the signal-tonoise ratio; various methods (averaging, principal component analysis, independent component analysis, subtraction methods, projection operators, etc.) can be used to extract the part of the response that is due to brain activity related to the experimental condition instead of unrelated events such as instrumental noise, background cerebral activity, muscle activation, or the decay of TMS-induced electrode polarization.

Among methods to stimulate the intact brain, TMS is unique in that it activates all its primary target neurons at the same time. When the rising phase of the magnetic field is over, the induced current density is reversed; the average induced electric field is zero. The average induced electric current may differ slightly from zero if the tissue conductivity changes during the brief pulse. Therefore, one expects no significant long-term effects from the TMS other than the initiation of action potentials and whatever processes these may, in turn, elicit. This is also true for EEG: the induced current being over within a fraction of a millisecond, the TMS-evoked brain response is probably due to cellular mechanisms that were triggered by the pulse, not by the pulse itself or remnants of accumulated charge. The latter is possible in principle if brain tissue contains rectifying and charge-storage elements for the induced current but no evidence appears to exist for such mechanisms to play a role in TMS.

The initial submillisecond synchrony, however, is soon lost because of conduction from the site of stimulation to the first synapses and further along the neuronal network initiating a cascade of serial and parallel effects. The bestknown such effect is the motor-evoked potential (MEP), measured from peripheral muscles after TMS applied to the motor cortex. Because of the activation of inhibitory cells in addition to excitatory ones, the stimulated cortex appears to assume an inhibitory state for a period of $100 \mathrm{~ms}$ or more, at least in the motor cortex. This is known as the cortical silent period (CSP, Merton 1951; Abbruzzese and Trompetto 2002), which is evidenced by a period of quiescence of EMG activity following each MEP; the effect is most clearly seen when the subject is contracting the target muscle. However, local inhibition is not the only major effect: before the CSP has time to start, pyramidal cells are activated and send their signals to neighboring and distant parts of the brain and towards the periphery. From estimates of synaptic delays and axonal transmission velocities one can predict how soon after the pulse other brain regions can be activated. Such estimates might serve as prior information that may prove to be useful in sorting out artifacts from brain signals.

In addition to standard evoked responses, TMS may also trigger oscillatory activity (Paus et al. 2001; Fuggetta et al. 2005; Rosanova et al. 2009) or perturb ongoing rhythms (Rosanova et al. 2009), eliciting, e.g., event-related synchronization (ERS) or desynchronization (ERD) (Pfurtscheller and Lopes da Silva 1999) or more complicated phenomena. The measured signals give significant information about the functional state of the brain. For example, effects of vigilance, pharmaceuticals, task performance as well as previous magnetic stimulation on TMS-evoked EEG responses have been reported. EEG may also serve as a safety monitor of the overall brain activity in, e.g., epilepsy or stroke patients.

The recording and analysis of TMS-evoked responses follows for the most part the same logic and principles as those of any other evoked response data. Here we discuss mainly the unique features of TMS-EEG; the general principles of EEG have been thoroughly documented elsewhere (e.g., Regan 1989).

\section{Mechanisms of TMS-Evoked EEG Generation}

The TMS-induced electric field depends on the relative location and orientation of the coil and the head, the head's large-scale structure and the local details of conductivity. As the cellular-scale structure is always unknown, only a coarse-grained electric field can be calculated, for example using a spherical model fitted to the local curvature of the intracranial cavity. The activation of the cortex depends on the orientation of the induced field with respect to the sulci, the optimal direction being perpendicular to the cortical surface (Fox et al. 2004). It has to be noted that both coil orientation and TMS intensity may affect the relative activation of different neuronal populations (e.g., pyramidal cells vs. inhibitory interneurons).

A curious and in some cases important observation regarding TMS-evoked EEG responses was pointed out by 
Ilmoniemi and Karhu (2008). At least in the spherically symmetric approximation of the head, no EEG signals would be observed if the TMS-triggered neuronal currents would be linearly related to and in the same direction as the TMS-induced field. Therefore, some significant features of the neuronal response may go unnoticed or at least be reduced in amplitude in the EEG, in particular if a round coil with its cylindrically symmetric induced field pattern is used. Since the cortical response to TMS in fact is highly nonlinear, this problem is probably not essential when focal figure-of-eight coils are used: the activation they produce is limited to such a small area that EEG signal cancellation is to a large extent avoided.

In contrast to the high variability of motor evoked potentials (MEPs), the TMS-evoked EEG averaged responses are generally highly reproducible, provided that the delivery and targeting of TMS is well controlled and stable from pulse to pulse and between experiments. Lioumis et al. (2009) reported high reproducibility of TMS-evoked EEG deflections with correlation factor exceeding 0.83 for all components up to $200 \mathrm{~ms}$ post-stimulus. Kičić (2009) and coworkers have identified several components of the EEG response to single-pulse TMS in the motor cortex (see Fig. 1): N15 (negative EEG deflection peaking approximately $15 \mathrm{~ms}$ post-stimulus), P30 (positive), N45, P55, $\mathrm{N} 100, \mathrm{P} 180$ - a response structure that is in agreement with earlier findings (Komssi et al. 2002, 2004; Nikouline et al. 1999; Paus et al. 2001; Bender et al. 2005; Massimini et al. 2005; Esser et al. 2006). However, these components are not universal; in addition to interindividual differences, the responses depend on the exact coil location (Komssi et al. 2002) and orientation, on the state of the cortex (Nikulin

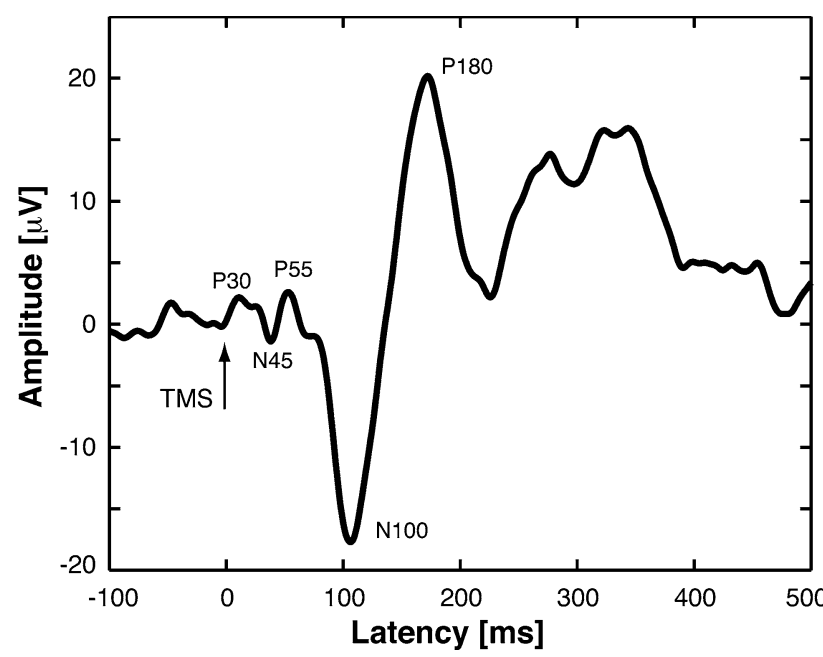

Fig. 1 TMS-evoked EEG response from the motor cortex: singlechannel response in the vicinity of the stimulated cortical site. The names of the components relate to the polarities and latencies. The structure and latencies of the peaks may vary slightly between subjects and measurements et al. 2003), and on the vigilance of the subject (Massimini et al. 2005). The latter authors found that in non-REM dreamless sleep, the EEG response is even stronger than during wakefulness but that the response has fewer deflections and is spatially limited to the vicinity of the stimulated spot. Nikulin et al. (2003) found that the N100 response from the motor cortex is smaller during a motor task than during rest. Komssi et al. (2004), studying the dependence of the EEG response on TMS intensity, concluded that brain activation elicited by TMS depends on the distribution of membrane potentials at the time of stimulus: only weak pulses are required to activate neurons that are already close to the firing threshold.

EEG is not very sensitive to action potentials because of their symmetric current distribution and short duration. Thus, it is believed that postsynaptic currents generate most of the EEG. The initial TMS-evoked response, although difficult to measure without artifact contamination, appears to result from the activation of the target area whereas later deflections are partially due to activity triggered by axonally conducted signals. How the signals are transmitted depends strongly on the state of the firing of diffuse neuromodulatory systems of the brain (Massimini et al. 2005; Kähkönen et al. 2001), but also on local activation at the time of stimulus delivery (Romei et al. 2008a). The understanding of the TMS-evoked activity that is elicited at sites distant from the TMS target can benefit from knowledge of the anatomical connectivity of the brain as seen by diffusion tensor imaging (DTI; Le Bihan et al. 2001).

\section{The Challenge of Combining TMS and EEG}

If a standard EEG system is used together with TMS, it can take hundreds of milliseconds for the amplifiers to recover from the large induced electric signal, which may have saturated one or several of the amplifiers. Furthermore, even after the recovery, recharging of the TMS device for the next pulse may cause a significant artifact. Even in the most advanced TMS-compatible EEG systems, the recharging of an EEG-incompatible TMS device usually results in an artifactual peak appearing at variable latencies in the signal, depending on the power of the subsequent TMS pulse (Veniero et al. 2009). This problem can be avoided by using monophasic devices, or by inserting a recharging delay circuit in the stimulation instruments.

If the TMS coil is in contact with the electrodes, these may move with the result of ruining the measurement. In addition, standard electrodes are heated by repeated stimuli with a risk of skin burns. The loud click sound from TMS and skin sensations produce auditory and somatosensory responses, respectively, that may mistakenly be interpreted 
as TMS-evoked brain activity. One must also remember that reliable TMS-EEG studies require one to control accurately for the location of the TMS coil: a movement of $5 \mathrm{~mm}$ between conditions may cause a large change in the evoked signals (Komssi et al. 2002). This is particularly important in follow-up studies.

Standard EEG systems are often sufficient in studies where one wants to monitor EEG prior to the pulse or to measure changes of oscillatory activity several hundred milliseconds afterwards. Even then, one must make sure that the electrodes are not heated too much and that the TMS electronics does not cause artifacts.

Even with the state-of-the-art EEG systems that claim to have eliminated the electromagnetic artifact, the recording of TMS-evoked responses remains a challenge. Therefore, it is necessary to systematically analyze the design, execution, and data analysis of TMS-EEG recordings.

For simple monitoring of brain background activity or variations in response amplitude, a few electrodes usually suffice, but to determine source distributions in the brain, high-resolution recordings with some 50-100 recording channels are needed. There is little evidence of major gains in determining the source distribution if the number of electrodes is increased much above 100; the law of diminishing gains is certainly valid here.

\section{Electrode Type}

The purpose of the electrode is to allow the measurement of the electric potential on the skin. The optimal electrode should satisfy numerous physical requirements to operate in harsh electromagnetic environment of the TMS: it has to have small enough diameter not to overheat or to be affected too much by the forces due to induced currents, must be coated with suitable surface material for the best interface with the skin, and designed appropriately to meet all these requirements at the same time. Suitable electrodes to record TMS-evoked EEG activity are small $\mathrm{Ag} / \mathrm{AgCl}$ pellet electrodes (e.g., Roth et al. 1992; Virtanen et al. 1999; Ives et al. 2006); these are now used in most commercial TMS-EEG systems.

Roth et al. (1992) found that the temperature of the electrode is elevated in proportion to the square of TMS intensity and the square of the electrode diameter while its thickness had no effect. Let us consider a thin silver disk with radius $r=5 \mathrm{~mm}$, conductivity $\sigma=6.2 \times 10^{7} \mathrm{~S} / \mathrm{m}$, density $\rho=10490 \mathrm{~kg} / \mathrm{m}^{3}$, and specific heat $c=235 \mathrm{~J} /$ $(\mathrm{kg} \mathrm{K})$. Let us assume a bipolar pulse with the magnetic field perpendicular to the disk, $B=B_{0} \sin (\omega t)$, where $B_{0}=1 \mathrm{~T}$ and $\omega=2 \pi / \Delta t=21 \mathrm{kHz}$ if the duration of the pulse is $\Delta t=0.3 \mathrm{~ms}$. For other orientations of the field, heating is less intense. TMS induces circular currents in the disk. The voltage around the electrode rim is equal to the time derivative of the magnetic flux through the disk: $V=\pi r^{2} d B / d t=\pi r^{2} B_{0} \omega \cos (\omega t)$; the induced electric field is $E=V /(2 \pi r)=(r / 2) B_{0} \omega \cos (\omega t)$. The induced current density, $J=\sigma E$, causes ohmic heating at the rate of $P=J^{2} / \sigma=\sigma\left(r^{2} / 4\right) B_{0}^{2} \omega^{2} \cos ^{2}(\omega t)$. The average of $\cos ^{2}(\omega t)$ being $1 / 2$, the energy density per pulse is $\Delta W=\sigma\left(r^{2} /\right.$ 8) $B_{0}^{2} \omega^{2} \Delta t$. The temperature rise is then $\Delta T=\Delta W /$ $c \rho=\left(\pi^{2} / 2\right)\left(\sigma r^{2} / c \rho\right) B_{0}^{2} / \Delta t=10 \mathrm{~K}$ with the materials and pulse values that were assumed. Since silver is a good conductor of heat, the less heated center of the electrode diminishes the temperature rise by $50 \%$. Thus, a single pulse would heat the electrode by $5 \mathrm{~K}$. It is then possible to exceed with just a few pulses the limit of $41^{\circ} \mathrm{C}$ imposed by safety standards for medical equipment (IEC-601); somewhat higher temperatures may cause pain or even inflict skin burns. Temperature variations can also give rise to artifacts in the EEG signal. Standard disk electrodes are therefore unacceptable for use with TMS.

There are basically two ways to reduce eddy-current heating and forces on electrodes: reduction of current-loop areas or reduction of conductivity of the electrode. Virtanen et al. (1999) used a slit in an annulus-shaped electrode and found that heating was reduced by an order of magnitude. The problem can also be avoided by using small pellet electrodes. The other possibility is to use electrode material with a low value of conductivity. Thut et al. (2005) have used conductive plastic electrodes (Plastics One, Roanoke, VA, USA) coated with silver epoxy to create an $\mathrm{Ag}-\mathrm{AgCl}$ surface for ensuring high-quality recordings.

It is generally recommended that the electrode impedance (resistance) is below $5 \mathrm{k} \Omega$. There are two reasons for such a recommendation. One is thermal voltage noise (Johnson noise) in any resistance $R$; the noise spectrum is uniform (white) and its root-mean-square (RMS) amplitude in a $1-\mathrm{Hz}$ frequency band is $V_{\mathrm{n}}=2(k T R)^{1 / 2}$, where $T$ is the temperature and $k$ is Boltzmann's constant. However, Johnson noise does not usually limit the sensitivity of lowfrequency EEG recordings as long as the electrode resistance is at most hundreds of $\mathrm{k} \Omega$. Another, more important reason for a low resistance in TMS studies is that artifacts from electrode movements or polarization are smaller when the contact resistance is low.

To achieve low electrode impedance, the skin under the electrode has to be cleaned, for example by alcohol; scrubbing the skin before applying electrode paste helps reduce the resistance further. As resistances may change during the measurement, it is recommended that the contacts be checked at least during long recording sessions. Further improvements in electrode contacts are possible: Julkunen et al. (2008) demonstrated that puncturing the epithelium under the scalp electrodes can reduce the TMSinduced artifact. 
Amplifiers

The first obstacle one has to tackle when recording TMSevoked brain responses is the large voltages induced in the loops formed by the combination of electrode leads, amplifier circuits, and the head. In each loop, the voltage is simply the time derivative of the magnetic flux threading the loop. This problem can be minimized by using twisted wire or coaxial cables and by compensating any remaining loops with oppositely oriented loops, but satisfactory compensation is difficult for a multichannel system. If a loop of just $1 \mathrm{~cm}^{2}$ remains (but note that typical loops are 1-2 orders of magnitude larger) and the TMS field increases from 0 to 1 tesla in $0.1 \mathrm{~ms}$, the induced voltage is $1 \mathrm{~V}$, orders of magnitude above the microvolts we measure from the brain.

As pointed out above, for many purposes one can use amplifiers that do not entirely avoid the EEG artifact; instead, they recover from the pulse after a delay, which is typically a large fraction of a second (Fuggetta et al. 2005; Iramina et al. 2002; Izumi et al. 1997). Iramina et al. (2003) designed an amplifier that includes an attenuator and a semiconductor switch. In one sample-and-attenuate stage, it actively attenuates the signal during the full duration of the TMS pulse. The EEG amplifiers were switched off $10 \mathrm{~ms}$ before the TMS pulse and switched back on $1 \mathrm{~ms}$ after; the TMS artifact remained for approximately $10 \mathrm{~ms}$ post-stimulus.

An effective way to deal with the electromagnetic artifact was developed by Virtanen et al. (1999). Their 60channel EEG system (later commercialized by Nexstim Oy, Helsinki, Finland) is made TMS-compatible by gaincontrol and sample-and-hold circuits that prevent the strong artifact from being passed along the amplifier circuits. The blocking is triggered externally so that it begins immediately before the TMS pulse.

After the signals are high-pass filtered $(f>0.1 \mathrm{~Hz})$ and amplified, they are light-intensity modulated and transferred to a light receiver unit with optical fibres. After that, the analog signals are low-pass filtered, with cut-off frequency of $500 \mathrm{~Hz}$. The sampling rate during A/D conversion is $1450 \mathrm{~Hz}$. The gain of the first amplifier stage A1 (see Fig. 2) is reduced during the TMS pulse. Simultaneously, the semiconductor switch SW, following A1, opens the signal path during the TMS pulse: the input voltage of the second amplifier stage A2 drops to zero and the voltage over capacitor $\mathrm{C} 1$ remains constant. To block large voltage peaks before the optical isolator, the sampleand-hold circuit $\mathrm{S} / \mathrm{H}(\mathrm{A})$ latches the signal from $\mathrm{A} 2$ prior to the TMS pulse and keeps the output at this level during the pulse. $\mathrm{S} / \mathrm{H}(\mathrm{B})$, located in the non-isolated section of the amplifier, prevents any residual from the stimulus artifact from being stored in the subsequent filters (FLT). To keep the differential input voltage of the preamplifier A1 in the linear operating range, the signal in the positive input terminal $\mathrm{V}_{\text {in }}+$ is limited to $\pm 9 \mathrm{~V}$ (LIM), and the voltage between the negative terminal $\mathrm{V}_{\text {in }}-$ and the amplifier ground is kept smaller than $\pm 1 \mathrm{~V}$ by attaching the reference and ground electrodes close to each other. If the voltage exceeds these values, the $20-\mathrm{k} \Omega$ resistors $\mathrm{R} 1$ and R2 limit the current to a safe level in accordance with standards. The sample-and-hold circuit $\mathrm{S} / \mathrm{H}(\mathrm{B})$ is controlled by the Hold(B) signal, which is activated about $50 \mu$ s before the TMS pulse and is released after the pulse (e.g., $2.5 \mathrm{~ms}$ later). The sample-and-hold time is called 'the gating period'.

Ives and coworkers (Ives et al. 1998, 2006; Thut et al. 2003a, b, 2005) have presented an amplifier with a limited slew rate so that the electronics is not saturated by the pulse. The bandwidth is limited by the design to about $90 \mathrm{~Hz}$, but this is not a serious limitation for most studies. The system does not eliminate the electromagnetic artifact completely, but since the electronics remains continuously operational, the artifact can be removed by forming the difference between responses in two conditions. The drawback in this arrangement is the requirement of
Fig. 2 Block diagram of the TMS-compatible EEG amplifier by Virtanen et al. (1999), capable of recording the EEG responses to single TMS pulses after just a few milliseconds

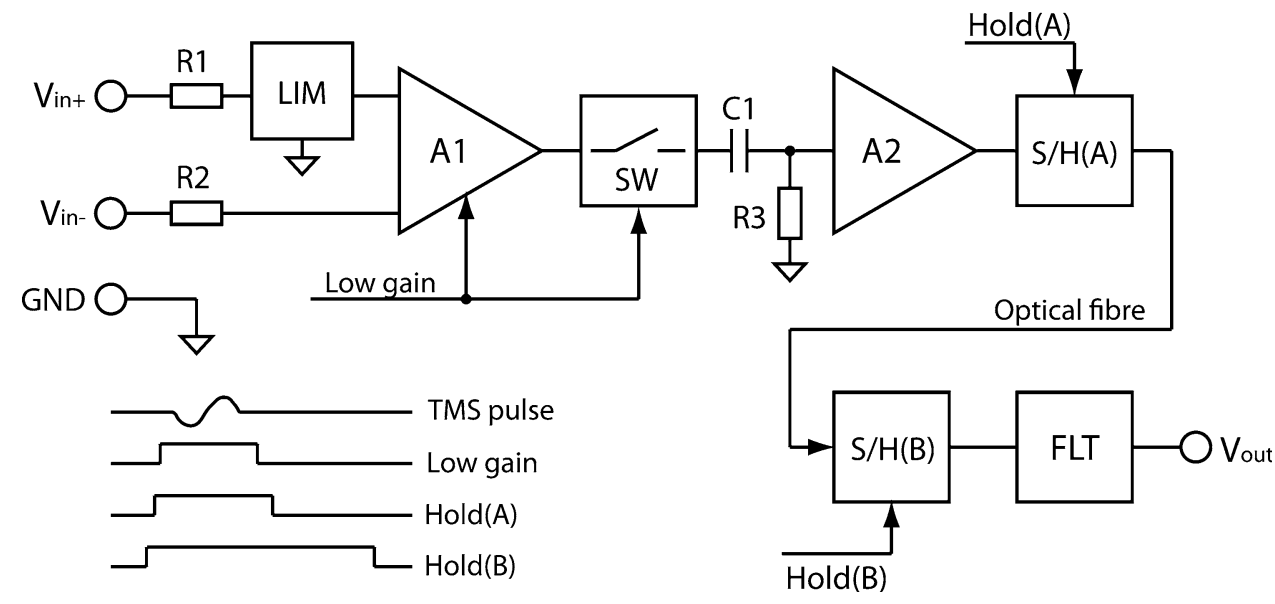


baseline or control measurements and the increased noise level due to the combination of the two signals.

Veniero et al. (2009) studied artifacts in the EEG signal induced by TMS using BrainAmp amplifiers (BrainProducts $\mathrm{GmbH}$, Munich, Germany). This EEG system allows one to adjust the sensitivity and operational range to match the applied TMS strength; in this case, the sensitivity of $100 \mathrm{nV} /$ bit was used. A continuous recording mode without the use of sample-and-hold circuits was possible. The recorded signals revealed artifacts produced by the magnetic pulse lasting approximately $5 \mathrm{~ms}$ following the TMS onset, after which the signals appeared to have recovered to the baseline level.

\section{Remaining Artifacts}

In addition to the induced artifact, TMS electronics may disturb EEG recordings by capacitive coupling or by ground-loop interference. Even when the electromagnetic artifact is dealt with successfully, other artifact problems remain. In the following, these are dealt with one by one.

\section{Eye Movements}

There is a steady potential of several millivolts across each eyeball (cornea has a positive potential with respect to the opposite side of the eye; eye movements therefore cause a transient potential at the vertex. This potential can be large compared to the EEG signal. A voluntary downward rotation of the eye for 10 degrees produces a negative potential of about $50 \mu \mathrm{V}$ at the vertex (Regan 1989). Some subjects perform systematic eye movements during the experimental sessions, for example synchronized with the preparatory interval. TMS can also trigger eye movements or blinks; these can be due to stimulation of eye-controlling brain areas or nerves innervating eye muscles or due to a startle effect caused by the sound of TMS or by scalp sensation from TMS. A solution to these problems is to monitor blinks and the movement of eyes with electrodes placed near them.

\section{Muscle Activity}

Superficial muscles on the head that are close to the EEG electrodes can cause strong artifacts in the recorded EEG signal, lasting up to about $30 \mathrm{~ms}$. Scalp muscles may be activated by TMS pulses, or they may contract for other reasons. The muscle artifact results from the depolarization of the muscle fibers (Paus et al. 2001). Most likely to be activated are the neck, the mastication, facial (Friedman and Thayer 1991), frontal, temporal, or masseter muscles, depending on the placement of the TMS coil. The muscle artifact can be reduced by moving or reorienting the coil, by reducing TMS intensity or by a combination of these. Sometimes even a small reduction in TMS intensity, to $90 \%$ of MT or less, may help significantly; at such intensities, EEG responses are still readily observable (Komssi et al. 2004, 2007; Kähkönen et al. 2005).

\section{Electrode Movement}

When a polarizable electrode is in contact with an electrolyte, a double layer of charge forms at the interface. If the electrode is moved with respect to the electrolyte, this movement mechanically disturbs the distribution of charge at the interface and results in a momentary change of the potential until equilibrium is re-established. This potential is known as the motion and movement artifact and can be a substantial source of disturbances in EEG. One should be very careful and avoid any contact with electrodes with the TMS coil; the vibration due to the pulse and any reaction by the experimenter (if coil is held by hand) may cause the electrode motion artifact (see Virtanen et al. 1999; Kähkönen et al. 2001, 2003). For example, a load of 500 grams on a skin-electrode interface has been reported to produce a change of about $5 \mathrm{mV}$ in electric potential (Tam and Webster 1977). Displacement of electrodes can be caused by the TMS coil touching them during stimulation, by muscle movements, or by electromagnetic forces in case of standard electrodes. The sensitivity of the electrodes to motion artifacts can be improved if the electrode-electrolyte interface is removed from a direct contact with the stimulating coil. Movement artifacts are in the frequency range of many bioelectric events (Geddes and Baker 1980), but filtering can be used with success.

\section{Electrode Polarization}

Electrode polarization is caused by electric currents between the electrolyte and the electrode. Theoretically, two types of electrodes are possible: perfectly polarizable and perfectly non-polarizable. Perfectly polarizable electrodes would be those in which no actual charge crosses the electrode-electrolyte interface when current is applied. The current across the interface is displacement current and the electrode behaves as it were a capacitor. In case of perfectly non-polarizable electrode, the current passes freely across the electrode-electrolyte interface. Real electrodes are somewhere between these extremes; the interface resembles a voltage source and a capacitor. If an electrode is polarized, it may take up to hundreds of milliseconds to return to equilibrium potential after the TMS pulse. Such a decay of the potential consists of one or more exponentially diminishing functions. Litvak et al. (2007) have dealt with 
this kind of artifacts by fitting exponential functions to the signal and by removing them.

\section{Coil Click and Somatic Sensation}

Electromagnetic forces within the coil give rise to a loud click (up to $120 \mathrm{~dB}$ ), which obviously activates the subject's auditory system and gives rise to an evoked potential that can be misinterpreted (Nikouline et al. 1999; Tiitinen et al. 1999; Bender et al. 2005). Good hearing protection helps, but is not usually sufficient. Even if the headphones would dampen the sound completely, some of it is conducted via the bones of the skull (Nikouline et al. 1999). Nikouline et al. (1999) showed that clicks, propagated both via air and via head bones, may evoke considerable auditory evoked potentials. Therefore, to guarantee complete elimination of the auditory response, masking sound must be used in addition to hearing protection (Fuggetta et al. 2005; Paus et al. 2001). To minimize the power of the masking noise, Massimini et al. (2005) applied noise that had the same frequency spectrum as the TMS clicks.

One also has to be aware of the fact that TMS-elicited scalp sensations (from muscle movements and direct sensory-neuron stimulation) produce evoked responses in the brain. Somatosensory evoked potentials (SEP) arising from the scalp are asymmetric, with the largest amplitude over the contralateral hemisphere (Bennett and Jannetta 1980; Hashimoto 1988), whereas the asymmetry of the TMSevoked EEG responses in most cases is reversed (Nikulin et al. 2003; Komssi et al. 2004; Kähkönen 2005; Kičić et al. 2008; Bikmullina et al. 2009; see Fig. 3). The latency of the N45 response coincides with that involving the conduction of a motor command to the hand muscles and the return of subsequent sensory afferent to the cortex (Tokimura et al. 2000). However, the potential pattern of N45 remains unchanged regardless of sub- or suprathreshold TMS intensities, strongly indicating that N45 is not generated by afferent input from peripheral muscles (Nikouline et al. 1999; Paus et al. 2001). It is important to keep in mind that somatosensory stimulation of the scalp involves activation of the trigeminal nerve; the resulting SEPs have the largest peak-to-peak amplitude $(<4 \mu \mathrm{V})$ in the first $80 \mathrm{~ms}$ (Bennett and Jannetta 1980; Hashimoto 1988); the later peaks are smaller $(<2 \mu \mathrm{V}, 100 \pm 200 \mathrm{~ms})$. Since the TMS-evoked EEG response has consistent alternating deflections starting at $15 \mathrm{~ms}$ post-stimulus, with the largest amplitudes being in the 100-200 ms interval, it is very difficult to evaluate the degree of contribution of somatosensory signals due to TMS-activated muscle movements (Nikouline et al. 1999). Investigators have come to the conclusion, however, that this is not a major problem (Paus et al. 2001; Nikouline et al. 1999; Nikulin et al. 2003).

\section{How to Perform a Successful TMS-Evoked EEG Measurement}

Recording EEG in the harsh electromagnetic environment of TMS is technically and methodologically challenging.
Fig. 3 Averaged responses evoked by TMS in one subject. The signals are arranged according to the layout of the electrodes (the view is from the top of the head, nose pointing upward). Prominent response amplitudes at latencies of approximately $50-100 \mathrm{~ms}$ are dominant in the vicinity of the stimulated point (denoted with ' $x$ '). Note the lateralization of responses: in the vicinity of the stimulated site, the amplitudes are the highest, attenuating with increasing distance from the coil

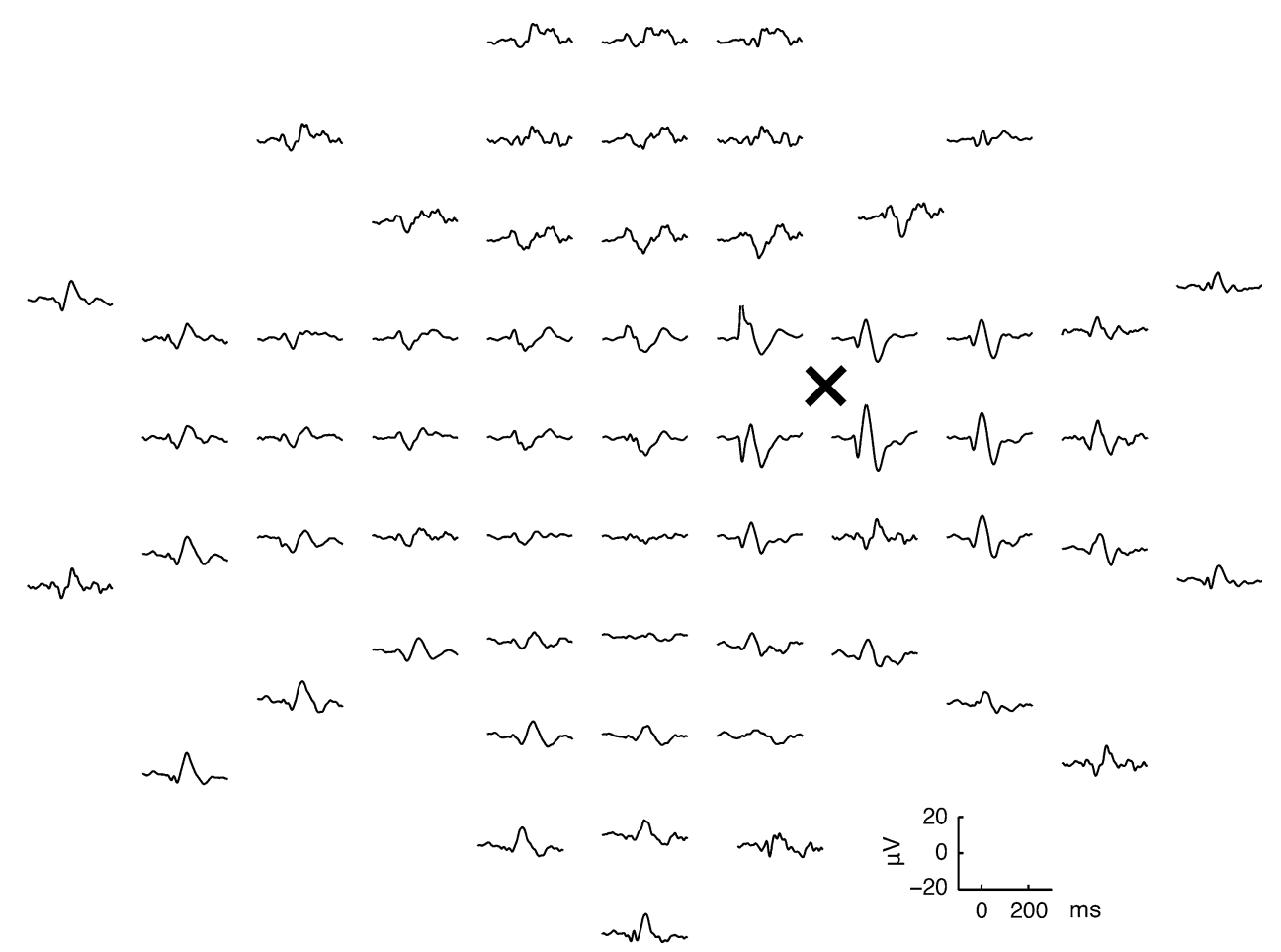


With multichannel EEG recordings, artifact removal and data analysis starts already at the acquisition time. Proper technological solutions for recording environment, electrodes, amplifiers, careful methodological approach, and suitable analysis methods should be used to eliminate the effects of the strong TMS pulse, which is potent enough to cause large and readily visible disturbances in the EEG.

\section{Subject Preparation}

Apart from the technical equipment itself, the following critical factors should be considered in order to obtain high-quality EEG recordings. (1) A thorough preparation of the skin under the electrodes. Ideally, the impedances of the electrodes should be kept below $5 \mathrm{k} \Omega$; a relatively small amount of gel should be applied in order to avoid 'bridging' between the electrodes. (2) The TMS coil should be immobilized with respect to the head, e.g., by pressing it against the head; this must be done without touching or even indirectly affecting the electrodes during the measurement.

\section{Use of Neuronavigation for Target Selection}

Komssi et al. (2002) showed that 10 -mm shifts in coil location results in large changes in the TMS-evoked EEG pattern. Such findings have taught us two things: (1) Highly location-specific information can be obtained with TMSEEG; (2) Proper interpretation of the results and their comparison within and between individuals require accurate control and knowledge of the cortical site that is stimulated. Traditionally, good control of the location has been possible when targeting the motor cortex; the stimulator coil has been adjusted so that the motor response is maximized or motor threshold is minimized. A functional targeting accuracy of a few $\mathrm{mm}$ has been possible. Another area that has a signature output is the visual cortex: TMS over the visual cortex can induce illusory visual percepts (phosphenes, Epstein et al. 1996; Silvanto et al. 2007; Romei et al. 2007, 2008a). For other areas of the cortex that are behaviorally silent (Penfield 1958), the precise targeting generally requires a navigation technique that computes from the measured coil location and orientation the induced electric field distribution in the brain and shows this superimposed on the subject's own MR image. One can then move the peak of the induced field to the target.

Navigated TMS (nTMS; also called navigated brain stimulation or NBS) targeting system is based on individual structural magnetic resonance images (MRI) and provides precise information regarding cortical surface anatomy and/or lesions in individual patients. Using such a system, the cortical target as well as coil position and orientation can be monitored in real time throughout the sessions. Such a device allows one to determine the locations of the EEG electrodes, the orientation and location of the coil, and the induced electric field for every TMS pulse (e.g., Bikmullina et al. 2009; Raij et al. 2008). These recorded parameters can be recalled to reproduce the location and orientation (direction and angle) of the coil in subsequent stimulation sessions. In TMS-evoked EEG, accurate reproducibility of the stimulation parameters is essential for any comparative or longitudinal studies. However, it is important to emphasize that targeting of TMS according to anatomical brain structures does not always lead to the stimulation of identical functional areas in different subjects because of interindividual differences in structure-function relationships, and thus affect the accuracy of general indices of cortical responsiveness (Casali et al. 2010).

The latest technological incarnation in navigated TMS is based on a subject's individual fMRI data for the respective cognitive function. Based on individual imaging results, TMS is subsequently used to probe whether the identified task-correlated activities in the fMRI-localized areas are necessary for successful task performance (Andoh et al. 2006; Sack et al. 2006; Thiel et al. 2005). Sparing et al. (2008) and coworkers have addressed this method by evaluating the accuracy and efficiency of different localization strategies for TMS-based primary motor mappings, and found the highest precision with fMRI-guided stimulation, which was accurate at the millimeter level. The same result was obtained by Sack et al. (2009), who revealed a systematic difference between tested approaches, with the individual fMRI-guided TMS neuronavigation yielding the strongest behavioral effect. This suggests that the different coil positioning approaches do not necessarily differ qualitatively in their TMS-induced effects, but in the magnitude of their respective effect sizes, and thus, in the number of participants required to reveal statistical significance of the observed TMS-induced changes. This approach accounts for inter-individual differences both in brain anatomy and in the functional architecture of the brain.

\section{Practical Issues During Measurements}

The use of the newest small pellet electrodes should be considered. Compared to the traditional larger electrodes, the pellet electrodes are less prone to interact with high magnetic field and therefore cause less movement artifacts and heating (e.g., Roth et al. 1992; Virtanen et al. 1999) and have by far better electrolyte contact with the scalp. These electrodes also nearly eliminate the DC shift that TMS pulses tend to produce.

The use of neuronavigation for targeting the TMS coil reduces muscle artifacts because errors in targeting are not over-compensated with increased TMS intensity. Muscle 
artifacts can sometimes be reduced by changing slightly the location or orientation of the coil (Ilmoniemi and Karhu 2008).

Bio-electromagnetic super-sensitive recordings should be done in an electromagnetically (EM) silent environment away from power lines and other EM disturbances (Simelius et al. 1995), and if necessary in a shielded room.

\section{Data Analysis}

\section{General Steps}

EEG data should be inspected epoch-by-epoch either visually or automatically together with the simultaneously recorded electro-oculogram (EOG). Each epoch with a considerable (above $100 \mu \mathrm{V}$ ) eye-blink signal should normally be rejected from further analysis. Also, epochs containing large artifacts from electromagnetic residuals or muscle activity (for example in the channels in the vicinity of the TMS coil) must be rejected. Following this procedure, the maximum rejection rate in a single session should remain reasonably low. If this is not possible, one must resort to more advanced methods that enable the separation of brain signals from artifacts. Such methods include signal-space projection, independent component analysis, modeling of sources and artifacts etc. If the analysis protocol includes data averaging, one has to end up in having several tens of epochs in each experimental condition. Importantly, if the analysis protocol comprises contrasting central (EEG) versus peripheral (EMG) manifestations of the phenomena under investigation (e.g., Kičić et al. 2008; Bikmullina et al. 2009), it is useful to bear in mind that if the EEG epoch is rejected, the corresponding EMG epoch must be rejected as well.

\section{Regions of Interest}

Most TMS-EEG investigations address the role or behavior of specific cortical areas (e.g., Raij et al. 2008) or hemispheres (e.g., Kičić et al. 2008) in the functional network under study. For that, a suitable analysis method is to select regions of interest (ROI) covered by a limited number of EEG channels. The ROIs for that purpose could be selected on the basis of the most pronounced TMS-evoked N100 response with the goal of addressing specific local excitability changes. If the channels were selected for the analysis after determining the individual peak locations of some of the components of the TMS-evoked EEG, the ROI would most probably shrink to 1-4 electrodes, giving thus very local information about cortical processing. If, on the other hand, the number of channels would be chosen on the basis of the extent of the potential pattern, the ROI might spread to more electrodes (10-15, e.g., Nikulin et al. 2003), giving more global hemispheric differences about the processing of interest. In this case, the local effect under investigation may lose statistical power. Optimal selection of a sufficient number of EEG channels in the definition of the ROI is recommended so as to keep focus on local excitability changes, but at the same time to be 'widespread' enough to capture the most pronounced EEG activity. For example, Tamas et al. (2003) showed that inhibition evoked by a distinct interneuronal population in spatially restricted postsynaptic compartments could locally and selectively modulate cortical excitability (see also Fitzgerald et al. 2009). Based on that finding, Bikmullina et al. (2009) selected for comparison ROIs of four EEG electrodes bilaterally with the goal of addressing interhemispheric differences in cortical processing of short-latency afferent inhibition (SAI) after unilateral afferent input.

\section{Data Interpretation}

\section{Topography of TMS-Evoked Responses}

A typical topographic plot of TMS-evoked EEG responses after stimulation of the right motor cortex is shown in Fig. 3. The usual purpose of such a measurement is to detect both local and distant effects of TMS: to measure both local excitability of the stimulated patch of the cortex and the spreading of TMS-evoked activity in a broader cortical network.

Figure 3 shows also that the overall response amplitudes are highest right under the coil, diminishing with increasing distance from the stimulation point. An important feature of TMS-evoked EEG topography is that even though only one cortical hemisphere was stimulated, bilateral EEG responses are evoked with different features. TMS-evoked activity spreads from the stimulation site ipsilaterally via association fibers and contralaterally via transcallosal fibers and to subcortical structures via projection fibers (Ilmoniemi et al. 1997; Komssi et al. 2002, 2004; Iwahashi et al. 2008; Verleger et al. 2009). Locally, within one hemisphere, an increased EEG activity can be seen in a number of neighboring electrodes, suggesting the spread of TMSevoked activity to anatomically interconnected cortical areas (Bohning et al. 2000; Fox et al. 1997; Ilmoniemi et al. 1997; Komssi et al. 2002; Paus et al. 1997, 2001; Siebner et al. 2000; Strafella et al. 2001).

\section{State Dependency of TMS-Evoked EEG Responses}

An important challenge in interpreting TMS results comes from the fact that the effects of brain stimulation spread from the target site ortho- and antidromically in the 
neuronal network (Bohning et al. 2000; Fox et al. 1997; Ilmoniemi et al. 1997; Komssi et al. 2002; Paus et al. 1997, 2001; Siebner et al. 2000; Strafella et al. 2001). Similarly, the stimulated area is influenced by connected areas. Because area-to-area modulation is often inhibitory, corticospinal excitability (e.g., as reflected by the magnitude of the descending volley elicited by a given cortical stimulus) does not necessarily increase with the general level of cortical activity (Matthews 1999). Furthermore, virtual lesions (areas where normal operation is disrupted) are not limited to the stimulated spot but distributed along the neuronal network (Silvanto and Pascual-Leone 2008). There is growing evidence also from stimulation of cortical areas other than the motor cortex that the impact of TMS on the EEG response is not determined by the properties of the stimulus alone, but also decisively by the initial state of the activated brain region (Amassian et al. 1989; Schürmann et al. 2001; Ramos-Estebanez et al. 2007; Silvanto et al. 2007; Silvanto and Muggleton 2008).

Based on experimental findings that spontaneous oscillations in brain activity occur in well-defined neural networks (Goldman et al. 2002; Leopold and Logothetis 2003; Laufs et al. 2006; Beckmann et al. 2005; Damoiseaux et al. 2006), Romei et al. (2008a) analyzed features of the EEGbased prestimulus spectrogram to test the hypothesis that fluctuations in neuronal activity has a functional significance (Romei et al. 2008b) and may account for the variability in neuronal or behavioral responses to physically identical stimuli, such as TMS. They showed a direct link between fluctuations in alpha $(8-14 \mathrm{~Hz})$ activity over posterior recording sites and visual cortex excitability, measured by the EEG while stimulating the visual cortex by means of TMS. The visual cortex was more excitable when alpha activity was low, and less excitable when it was high, leading to TMS-induced visual percepts (phosphenes) or no percepts, respectively. Since the individual posterior alpha-band power correlates with the individual threshold for eliciting illusory, TMS-induced phosphenes (Romei et al. 2008b), they provided further evidence for the state dependency of visual perception and suggested that the spontaneous fluctuations of neuronal activity in areas of the visual network occurring in the presence of the task do not only modulate perception, but may underlie a functional role.

\section{Benefits of TMS-EEG}

Better Insight in Cortico-Cortical and Interhemispheric Interactions

Beside assessment of the general state of the brain (Massimini et al. 2005; Kähkönen et al. 2001), concurrent
TMS and EEG have the potential to offer insights into how brain areas interact during sensory processing (Bikmullina et al. 2009; Kičić et al. 2008; Raij et al. 2008; Silvanto et al. 2006; Mochizuki et al. 2004), cognition (Bonnard et al. 2009), or motor control (Nikulin et al. 2003; Kičić et al. 2008). Furthermore, EEG as a measure of cortical activity after the TMS pulse gives us the possibility to study cortico-cortical interactions by applying TMS to one area and observe responses in remote, but interconnected areas, or, more generally, how activity in one area affects the ongoing activity in other areas (Mochizuki et al. 2004; Silvanto et al. 2006). EEG correlates of the role of the frontal eye field (FEF) in attentional selection were recently addressed by Taylor et al. (2007), hypothesizing that if TMS to FEF has direct effects on the visual cortex, these effects should also be visible in TMS-evoked EEG. Indeed, TMS of the right FEF caused a within-trial modulation of activity in the right visual cortex, evident as a protracted shift in the baseline of the event-related potential (ERP). As expected, none of the effects of FEF TMS either on visual activity or on oculomotor control occurred during TMS of a somatosensory control site. In a study of interhemispheric interactions during unilateral movements, Kičić et al. (2008) stimulated in separate sessions both the ipsi- and contralateral motor cortices. As the TMS-evoked N100 component was modulated selectively depending on whether the subject performed ipsi- or contralateral (to TMS) unilateral motor action, they demonstrated that the preparation and execution of unilateral movement is associated with bilateral changes in cortical excitability. Since only in the contralateral hemisphere these changes were associated with modulation of peripheral muscle MEP responses, they concluded that such dissociation implies that additional inhibitory mechanisms in the ipsilateral hemisphere were recruited in order to suppress its motor output. These findings illustrate the important contribution brought by methodological development of TMS-EEG into research of functional cortical connections, especially because the time course of the TMS effect on the EEG can be related to the time course of the ongoing cognitive processes.

\section{More Direct Assessment of Cortical Inhibitory Processes}

In analogy with cortical studies (Krnjević et al. 1966; Rosenthal et al. 1967), besides activating the large excitatory Betz cells, which are found in abundance in the motor cortex, TMS activates also the inhibitory interneurons: their post-synaptic effects appear to be represented as the TMS-evoked N100 component. This was originally suggested by Nikulin et al. (2003) and subsequently supported by several other groups (Bender et al. 2005; Bonato et al. 
2006; Kičić et al. 2008; Bikmullina et al. 2009; Bonnard et al. 2009). It is important to have in mind that inhibitory processes in deeper cortical layers produce surface-negative potentials (Caspers et al. 1980), which identifies the N100 as a potential with inhibitory origins (Bender et al. 2005; Nikulin et al. 2003). The N100 is reportedly the most pronounced, the most reproducible, and long-lasting component in response to motor cortical TMS (Paus et al. 2001; Nikulin et al. 2003; Bender et al. 2005; Massimini et al. 2005; Kähkönen and Wilenius 2007; Kičić 2009; Lioumis et al. 2009; Bonnard et al. 2009). In a recent TMS-EEG study, Bonnard et al. (2009) revealed EEG correlates of cortical mechanisms underlying the interaction between cognitive and motor function by showing the relationship between anticipatory change in cortical excitability (as revealed by the contingent negative variation, $\mathrm{CNV}$ ) and cortical inhibitory processes (as revealed by the TMSevoked N100 component). They demonstrated that when subjects prepare to resist a TMS-evoked movement, the anticipatory processes cause a decrease in the cortical excitability by increasing inhibitory processes. Bikmullina et al. (2009) studied with TMS-EEG the cortical mechanisms of the phenomenon of short-latency afferent inhibition (SAI), which is attenuation of upper-limb MEPs by TMS due to preceeding stimulation of peripheral digital nerves or the median nerve at wrist (Tokimura et al. 2000; Classen et al. 2000; Tamburin et al. 2001). They showed that the attenuation of MEPs is positively correlated with the amplitude attenuation of the N100 response, revealing thus that even small individual changes in peripheral activity are paralleled by changes in cortically probed excitability. They interpreted these findings through an interaction between two inhibitory processes, partially coinciding over time. The first inhibition, due to incoming peripheral electrical stimulus (SAI), is directed at pyramidal cells and should produce hyperpolarization of the neuronal membrane, thus leading to a decrease in the MEP. At the time when the second, TMS-induced, inhibition starts, the neurons are already hyperpolarized due to SAI, resulting in a smaller N100 amplitude.

\section{Deeper Understanding of Cortical Plasticity and Oscillations}

The great promise in the use of repetitive TMS (rTMS) in the clinical domain is the possibility for plastic reorganization of cortical circuitry. The effects of rTMS have for the most part been demonstrated as effects on peripherally measured MEPs, becoming significant after delivery of a large number of pulses (Quartarone et al. 2005), and lasting for about $30 \mathrm{~min}$ post-rTMS (Peinemann et al. 2004). A number of studies indicate that such effects result from changes in the cerebral cortex (e.g., Di Lazzaro et al. 2002a, b; Quartarone et al. 2005). Indeed, the cerebral contribution has been revealed by numerous studies (for a comprehensive reference list, see Thut and Pascual-Leone 2009). However, only a few studies have used TMSevoked EEG responses to directly demonstrate cortical effects due to rTMS (Esser et al. 2006; Van Der Werf and Paus 2006; Huber et al. 2008). Esser et al. (2006) elegantly transformed the classical protocol (Bliss and Lomo 1973) to a TMS-EEG experiment in order to directly demonstrate long-term potentiation (LTP). They assessed total EEG activity using the global mean-field power (GMFP) and demonstrated that EEG responses to single TMS pulses delivered to motor cortex are increased in amplitude following the rTMS, most profoundly at latencies of 15-50 ms. Interestingly, they found evoked activity being strongest in electrodes located over the left premotor cortex and explained that areas distant from the site of stimulation are activated indirectly as the TMS-induced perturbation propagates through excitatory long-range pathways. They suggested a rapid termination of activity in the motor cortex, while activity propagates to, and persists in premotor cortex, resulting in an overall larger EEG response at that site. These findings open up promising possibilities to use this technique to assess where in the cortex the potentiation (or depression) are induced.

Is addition to the common analysis of TMS-evoked EEG data in the time domain, there is an increasing number of reports that TMS can also alter the spectral content of the EEG signal. For example, TMS to M1 increases the power of the beta-frequency $(15-30 \mathrm{~Hz})$ cortical oscillations recorded from adjacent electrodes (Paus et al. 2001). On the other hand, the effect of M1 TMS on the alpha power $(8-13 \mathrm{~Hz})$ increases with the intensity of TMS (Fuggetta et al. 2005) and the number of pulses administered. This effect correlates also with the reduction in MEP size (Brignani et al. 2008). Based on a great interest in the functional role of oscillatory brain activity in specific frequency bands of human participants, this line of research is especially promising since TMS-induced EEG effects on resting subjects can be shown at surprisingly low TMS intensities, and in a fashion that varies with stimulation site, intensity and pharmacological challenge (Taylor et al. 2008; Brignani et al. 2008). Deeper understanding of brain oscillations is expected from combining knowledge on how oscillatory activity in specific frequency bands is related to distinct functions (Jensen et al. 2007; von Stein et al. 2000; Sauseng et al. 2005; Hanslmayr et al. 2007a; Jensen and Colgin 2007; Komssi and Kähkönen 2006) with data from TMS-EEG studies (for reviews, see Thut and Miniussi 2009; Komssi and Kähkönen 2006). An excellent example of such methodological synergy is the TMS-EEG study by Rosanova et al. (2009), where they perturbed different parts of the corticothalamic system and measured their 
natural frequencies. They showed that each corticothalamic module is normally tuned to oscillate at a characteristic frequency, thus indicating that the observed oscillations reflect the physiology and connectivity of the brain rather than the parameters of TMS. Importantly, Rosanova et al. demonstrated that the specific frequency of the response did not depend on TMS intensity, or activation threshold, but most likely depended on endogenous properties of the activated circuits, and concluded that electrically-recorded cortical rhythms triggered by TMS most likely reflect overall circuit properties at the level of cortical areas and connected thalamic/subcortical nuclei.

\section{Prospects for Clinical Applications}

It has been suggested that gamma synchrony is affected in schizophrenia (Light et al. 2006; Cho et al. 2006; Lee et al. 2003; Green and Nuechterlein 1999): there might be underlying alterations of thalamocortical circuits. Ferrarelli et al. (2008) investigated EEG responses to single-pulse TMS of the premotor cortex in schizophrenic patients. The investigators compared, within the same subjects, gammarange spontaneous EEG activity with gamma responses evoked by single-pulse TMS. Consistent with previous findings (Kissler et al. 2000; Yeragani et al. 2006), they found no differences in the spontaneous gamma activity in schizophrenia patients and healthy subjects. However, in the same patients, they found a prominent decrease of TMS-evoked gamma oscillation. Thus, TMS-EEG offers a direct means to detect underlying deficits by challenging the relevant brain circuits with phasic (single TMS pulses) stimuli that engage the gamma oscillations, even when the deficits are not detectable under tonic conditions (Ferrarelli et al. 2008).

Huber et al. (2007) took advantage of TMS-EEG to record changes in cortical responses to TMS before and after $5-\mathrm{Hz}$ repetitive stimulation in order to study slowwave activity (SWA) during sleep. Their results indicate that high-frequency rTMS conditioning over the motor cortex leads to a local increase in the amplitude of the TMS-evoked EEG components between 10 and $130 \mathrm{~ms}$, indicative of potentiation of premotor circuits, followed (during subsequent sleep) by a prominent local increase in SWA. They found that SWA (and presumably the need for sleep) is increased by events leading to synaptic potentiation and decreased by events leading to synaptic depression, and that their regulation can occur locally in cortical circuits (Tononi and Cirelli 2003, 2006). This opens up new possibilities for TMS-EEG as a clinical tool in sleep disorders and in sleep-quality assessment.

The detection of the natural frequencies with TMS-EEG described by Rosanova et al. (2009; see previous section) may also have diagnostic potential and clinical applications, as it opens up possibilities to map the natural frequency of different cortical areas in various neuropsychiatric conditions such as depression, epilepsy, or disorders of consciousness. Since natural frequencies reflect relevant circuit properties, TMS-evoked EEG may radically extend the window opened by peripherally evoked MEPs. Whereas TMS-MEP is limited to motor areas, TMS-EEG can access any cortical region (primary and associative) in any category of patients and may offer a straightforward and flexible way to detect and monitor the state of corticothalamic circuits.

\section{Remaining Challenges}

\section{Generation of Evoked Responses}

The ERP responses evoked by sensory stimuli are produced by neuronal activity associated with stimulus processing in a time-locked manner. They are extracted from ongoing brain activity and system noise by averaging epochs of activity evoked by a series of stimuli. ERs are presumably generated either independently of ongoing oscillatory brain activity or through a stimulus-induced reorganization of ongoing activity. In the literature, three different mechanisms for the genesis of ERs have been put forward, advocating that ERs (i) are additive to ongoing oscillations (Shah et al. 2004; Mäkinen et al. 2005; Mazaheri and Jensen 2006), (ii) may result from a phase resetting of ongoing oscillations (Sayers et al. 1974; Makeig et al. 2002; Fell et al. 2004; Hanslmayr et al. 2007b), or (iii) may arise from modulation of the mean amplitude of ongoing activity, a phenomenon called baseline shift (Nikulin et al. 2007). These models are relevant to practically every electrophysiological measurement involving perceptual, cognitive, or motor activity. Therefore, putting the ER generation mechanisms in the context of TMS-evoked EEG responses is important for attempting to understand the functional schemes of neural circuits underlying both the neural information processing and human cognition in general.

\section{Conclusion}

The combination of TMS and EEG provides unique possibilities to study and map the excitability of the brain as well as its functional connectivity in a time-resolved manner. Furthermore, TMS-EEG offers the possibility to obtain detailed information about the state of the brain. This is possible, however, only if proper techniques and methods are used to deal with the electromagnetic, electrode-polarization, eye-blink, muscle, auditory and other artifacts. 
Even after all the techniques and precautions described in this paper have been properly taken into account and when the resulting signals look plausible or support one's favorite hypothesis, one has to be aware of the real possibility that the nice-looking EEG responses may in fact still be partially due to artifacts. Therefore, one usually needs control experiments or other means to ascertain that the signal we see actually originates in the brain and nothing but the brain.

Open Access This article is distributed under the terms of the Creative Commons Attribution Noncommercial License which permits any noncommercial use, distribution, and reproduction in any medium, provided the original author(s) and source are credited.

\section{References}

Abbruzzese G, Trompetto C (2002) Clinical and research methods for evaluating cortical excitability. J Clin Neurophysiol 19:307-321

Amassian VE, Cracco RQ, Maccabee PJ, Cracco JB, Rudell A, Eberle L (1989) Suppression of visual perception by magnetic coil stimulation of human occipital cortex. Electroencephalogr Clin Neurophysiol 74:458-462

Amassian VE, Cracco RQ, Maccabee PJ, Cracco JB (1992) Cerebellofrontal cortical projections in humans studied with the magnetic coil. Electroencephalogr Clin Neurophysiol 85:265-272

Andoh J, Artiges E, Pallier C, Riviere D, Mangin JF, Cachia A, Plaze M, Paillere-Martinot ML, Martinot JL (2006) Modulation of language areas with functional MR image-guided magnetic stimulation. Neuroimage 29:619-627

Barker AT, Jalinous R, Freeston IL (1985) Non-invasive magnetic stimulation of human motor cortex. Lancet 1:1106-1107

Beckmann CF, DeLuca M, Devlin JT, Smith SM (2005) Investigations into resting-state connectivity using independent component analysis. Philos Trans R Soc Lond B Biol Sci 360:1001-1013

Bender S, Basseler K, Sebastian I, Resch F, Kammer T, Oelkers-Ax R, Weisbrod M (2005) Electroencephalographic response to transcranial magnetic stimulation in children: Evidence for giant inhibitory potentials. Ann Neurol 58:58-67

Bennett MH, Jannetta PJ (1980) Trigeminal evoked potentials in humans. Electroencephalogr Clin Neurophysiol 48:517-526

Bikmullina R, Kičić D, Carlson S, Nikulin VV (2009) Electrophysiological correlates of short-latency afferent inhibition: a combined EEG and TMS study. Exp Brain Res 194:517-526

Bliss TV, Lomo T (1973) Long-lasting potentiation of synaptic transmission in the dentate area of the anaesthetized rabbit following stimulation of the perforant path. J Physiol 232:331-356

Bohning DE, Shastri A, Wassermann EM, Ziemann U, Lorberbaum JP, Nahas Z, Lomarev MP, George MS (2000) BOLD-f MRI response to single-pulse transcranial magnetic stimulation (TMS). J Magn Reson Imaging 11:569-574

Bonato C, Miniussi C, Rossini PM (2006) Transcranial magnetic stimulation and cortical evoked potentials: a TMS/EEG coregistration study. Clin Neurophysiol 117:1699-1707

Bonnard M, Spieser L, Meziane HB, de Graaf JB, Pailhous J (2009) Prior intention can locally tune inhibitory processes in the primary motor cortex: direct evidence from combined TMSEEG. Eur J Neurosci 30:913-923

Brignani D, Manganotti P, Rossini PM, Miniussi C (2008) Modulation of cortical oscillatory activity during transcranial magnetic stimulation. Hum Brain Mapp 29:603-612
Casali AG, Casarotto S, Rosanova M, Mariotti M, Massimini M (2010) General indices to characterize the electrical response of the cerebral cortex to TMS. Neuroimage 49:1459-1468

Caspers H, Speckmann EJ, Lehmenkuhler A (1980) Electrogenesis of cortical DC potentials. Prog Brain Res 54:3-15

Cho RY, Konecky RO, Carter CS (2006) Impairments in frontal cortical gamma synchrony and cognitive control in schizophrenia. Proc Natl Acad Sci USA 103:19878-19883

Classen J, Steinfelder B, Liepert J, Stefan K, Celnik P, Cohen LG, Hess A, Kunesch E, Chen R, Benecke R, Hallett M (2000) Cutaneomotor integration in humans is somatotopically organized at various levels of the nervous system and is task dependent. Exp Brain Res 130:48-59

Cracco RQ, Amassian VE, Maccabee PJ, Cracco JB (1989) Comparison of human transcallosal responses evoked by magnetic coil and electrical stimulation. Electroencephalogr Clin Neurophysiol 74:417-424

Damoiseaux JS, Rombouts SA, Barkhof F, Scheltens P, Stam CJ, Smith SM, Beckmann CF (2006) Consistent resting-state networks across healthy subjects. Proc Natl Acad Sci USA 103:13848-13853

Di Lazzaro V, Oliviero A, Berardelli A, Mazzone P, Insola A, Pilato F, Saturno E, Dileone M, Tonali PA, Rothwell JC (2002a) Direct demonstration of the effects of repetitive transcranial magnetic stimulation on the excitability of the human motor cortex. Exp Brain Res 144:549-553

Di Lazzaro V, Oliviero A, Mazzone P, Pilato F, Saturno E, Dileone M, Insola A, Tonali PA, Rothwell JC (2002b) Short-term reduction of intracortical inhibition in the human motor cortex induced by repetitive transcranial magnetic stimulation. Exp Brain Res 147:108-113

Epstein CM, Verson R, Zangaladze A (1996) Magnetic coil suppression of visual perception at an extracalcarine site. J Clin Neurophysiol 13:247-252

Esser SK, Huber R, Massimini M, Peterson MJ, Ferrarelli F, Tononi G (2006) A direct demonstration of cortical LTP in humans: a combined TMS/EEG study. Brain Res Bull 69:86-94

Fell J, Dietl T, Grunwald T, Kurthen M, Klaver P, Trautner P, Schaller C, Elger CE, Fernandez G (2004) Neural bases of cognitive ERPs: more than phase reset. J Cogn Neurosci 16:1595-1604

Ferrarelli F, Massimini M, Peterson MJ, Riedner BA, Lazar M, Murphy MJ, Huber R, Rosanova M, Alexander AL, Kalin N, Tononi G (2008) Reduced evoked gamma oscillations in the frontal cortex in schizophrenia patients: a TMS/EEG study. Am J Psychiatry 165:996-1005

Fitzgerald PB, Maller JJ, Hoy K, Farzan F, Daskalakis ZJ (2009) GABA and cortical inhibition in motor and non-motor regions using combined TMS-EEG: a time analysis. Clin Neurophysiol 120:1706-1710

Fox P, Ingham R, George MS, Mayberg H, Ingham J, Roby J, Martin C, Jerabek P (1997) Imaging human intra-cerebral connectivity by PET during TMS. Neuroreport 8:2787-2791

Fox PT, Narayana S, Tandon N, Sandoval H, Fox SP, Kochunov P, Lancaster JL (2004) Column-based model of electric field excitation of cerebral cortex. Hum Brain Mapp 22:1-14

Friedman BH, Thayer JF (1991) Facial muscle activity and EEG recordings: redundancy analysis. Electroencephalogr Clin Neurophysiol 79:358-360

Fuggetta G, Fiaschi A, Manganotti P (2005) Modulation of cortical oscillatory activities induced by varying single-pulse transcranial magnetic stimulation intensity over the left primary motor area: a combined EEG and TMS study. Neuroimage 27:896-908

Geddes LA, Baker LE (1980) Principles of applied biomedical instrumentation. Wiley, New York 
Goldman RI, Stern JM, Engel J Jr, Cohen MS (2002) Simultaneous EEG and fMRI of the alpha rhythm. Neuroreport 13:2487-2492

Green MF, Nuechterlein KH (1999) Cortical oscillations and schizophrenia: timing is of the essence. Arch Gen Psychiatry 56:1007-1008

Hämäläinen MS, Ilmoniemi RJ (1994) Interpreting magnetic fields of the brain: minimum norm estimates. Med Biol Eng Comput 32:35-42

Hanslmayr S, Aslan A, Staudigl T, Klimesch W, Herrmann CS, Bauml KH (2007a) Prestimulus oscillations predict visual perception performance between and within subjects. Neuroimage 37:1465-1473

Hanslmayr S, Klimesch W, Sauseng P, Gruber W, Doppelmayr M, Freunberger R, Pecherstorfer T, Birbaumer N (2007b) Alpha phase reset contributes to the generation of ERPs. Cereb Cortex $17: 1-8$

Hashimoto I (1988) Trigeminal evoked potentials following brief air puff: enhanced signal-to-noise ratio. Ann Neurol 23:332-338

Huber R, Esser SK, Ferrarelli F, Massimini M, Peterson MJ, Tononi G (2007) TMS-induced cortical potentiation during wakefulness locally increases slow wave activity during sleep. PLoS One 2:e276

Huber R, Määttä S, Esser SK, Sarasso S, Ferrarelli F, Watson A, Ferreri F, Peterson MJ, Tononi G (2008) Measures of cortical plasticity after transcranial paired associative stimulation predict changes in electroencephalogram slow-wave activity during subsequent sleep. J Neurosci 28:7911-7918

Ilmoniemi RJ (2009) What are evoked responses? In: 24th annual meeting of Japan Biomagnetism and Bioelectromagnetics Society, Kanazawa, Japan

Ilmoniemi R, Karhu J (2008) TMS and electroencephalography: methods and current advances. In: Wassermann EM, Epstein CM, Ziemann U, Paus T, Lisanby SH (eds) The oxford handbook of transcranial stimulation. Oxford University Press Inc., New York, pp 593-608

Ilmoniemi RJ, Virtanen J, Ruohonen J, Karhu J, Aronen HJ, Näätänen R, Katila T (1997) Neuronal responses to magnetic stimulation reveal cortical reactivity and connectivity. Neuroreport 8:35373540

Iramina K, Maeno T, Kowatari Y, Ueno S (2002) Effects of transcranial magnetic stimulation on EEG activity. IEEE Trans Magn 38:3347-3349

Iramina K, Maeno T, Nohaka Y, Ueno S (2003) Measurement of evoked electroencephalography induced by transcranial magnetic stimulation. J Appl Phys 93:6718-6720

Ives JR, Pascual-Leone A, Chen Q, Schlaug G, Keenan J, Edelman RR (1998) Experience and early findings using transcranial magnetic stimulation (TMS) during functional magnetic resonance imaging (fMRI) in humans. NeuroImage 7, Part 2 of 3:S33R

Ives JR, Rotenberg A, Poma R, Thut G, Pascual-Leone A (2006) Electroencephalographic recording during transcranial magnetic stimulation in humans and animals. Clin Neurophysiol 117: 1870-1875

Iwahashi M, Arimatsu T, Ueno S, Iramina K (2008) Differences in evoked EEG by transcranial magnetic stimulation at various stimulus points on the head. Conf Proc IEEE Eng Med Biol Soc 2008:2570-2573

Izumi S, Takase M, Arita M, Masakado Y, Kimura A, Chino N (1997) Transcranial magnetic stimulation-induced changes in EEG and responses recorded from the scalp of healthy humans. Electroencephalogr Clin Neurophysiol 103:319-322

Jensen O, Colgin LL (2007) Cross-frequency coupling between neuronal oscillations. Trends Cogn Sci 11:267-269

Jensen O, Kaiser J, Lachaux JP (2007) Human gamma-frequency oscillations associated with attention and memory. Trends Neurosci 30:317-324
Julkunen P, Pääkkönen A, Hukkanen T, Könönen M, Tiihonen P, Vanhatalo S, Karhu J (2008) Efficient reduction of stimulus artefact in TMS-EEG by epithelial short-circuiting by minipunctures. Clin Neurophysiol 119:475-481

Kähkönen S (2005) MEG and TMS combined with EEG for mapping alcohol effects. Alcohol 37:129-133

Kähkönen S, Wilenius J (2007) Effects of alcohol on TMS-evoked N100 responses. J Neurosci Methods 166:104-108

Kähkönen S, Kesäniemi M, Nikouline VV, Karhu J, Ollikainen M, Holi M, Ilmoniemi RJ (2001) Ethanol modulates cortical activity: direct evidence with combined TMS and EEG. Neuroimage 14:322-328

Kähkönen S, Wilenius J, Nikulin VV, Ollikainen M, Ilmoniemi RJ (2003) Alcohol reduces prefrontal cortical excitability in humans: a combined TMS and EEG study. Neuropsychopharmacology 28:747-754

Kähkönen S, Komssi S, Wilenius J, Ilmoniemi RJ (2005) Prefrontal TMS produces smaller EEG responses than motor-cortex TMS: implications for rTMS treatment in depression. Psychopharmacology (Berl) 181:16-20

Kičić D (2009) Probing cortical excitability with transcranial magnetic stimulation. Ph.D. Thesis. Helsinki University of Technology, Espoo

Kičić D, Lioumis P, Ilmoniemi RJ, Nikulin VV (2008) Bilateral changes in excitability of sensorimotor cortices during unilateral movement: combined electroencephalographic and transcranial magnetic stimulation study. Neuroscience 152:1119-1129

Kissler J, Müller MM, Fehr T, Rockstroh B, Elbert T (2000) MEG gamma band activity in schizophrenia patients and healthy subjects in a mental arithmetic task and at rest. Clin Neurophysiol 111:2079-2087

Komssi S, Kähkönen S (2006) The novelty value of the combined use of electroencephalography and transcranial magnetic stimulation for neuroscience research. Brain Res Rev 52:183-192

Komssi S, Aronen HJ, Huttunen J, Kesäniemi M, Soinne L, Nikouline VV, Ollikainen M, Roine RO, Karhu J, Savolainen S, Ilmoniemi RJ (2002) Ipsi- and contralateral EEG reactions to transcranial magnetic stimulation. Clin Neurophysiol 113:175-184

Komssi S, Kähkönen S, Ilmoniemi RJ (2004) The effect of stimulus intensity on brain responses evoked by transcranial magnetic stimulation. Hum Brain Mapp 21:154-164

Komssi S, Savolainen P, Heiskala J, Kähkönen S (2007) Excitation threshold of the motor cortex estimated with transcranial magnetic stimulation electroencephalography. Neuroreport 18:13-16

Krnjević K, Randić M, Straughan DW (1966) An inhibitory process in the cerebral cortex. J Physiol 184:16-48

Laufs H, Holt JL, Elfont R, Krams M, Paul JS, Krakow K, Kleinschmidt A (2006) Where the BOLD signal goes when alpha EEG leaves. Neuroimage 31:1408-1418

Le Bihan D, Mangin JF, Poupon C, Clark CA, Pappata S, Molko N, Chabriat H (2001) Diffusion tensor imaging: concepts and applications. J Magn Reson Imaging 13:534-546

Lee KH, Williams LM, Breakspear M, Gordon E (2003) Synchronous gamma activity: a review and contribution to an integrative neuroscience model of schizophrenia. Brain Res Brain Res Rev 41:57-78

Leopold DA, Logothetis NK (2003) Spatial patterns of spontaneous local field activity in the monkey visual cortex. Rev Neurosci 14:195-205

Light GA, Hsu JL, Hsieh MH, Meyer-Gomes K, Sprock J, Swerdlow NR, Braff DL (2006) Gamma band oscillations reveal neural network cortical coherence dysfunction in schizophrenia patients. Biol Psychiatry 60:1231-1240

Lioumis P, Kičić D, Savolainen P, Mäkelä JP, Kähkönen S (2009) Reproducibility of TMS-evoked EEG responses. Hum Brain Mapp 30:1387-1396 
Litvak V, Komssi S, Scherg M, Hoechstetter K, Classen J, Zaaroor M, Pratt H, Kahkonen S (2007) Artifact correction and source analysis of early electroencephalographic responses evoked by transcranial magnetic stimulation over primary motor cortex. Neuroimage 37:56-70

Makeig S, Westerfield M, Jung TP, Enghoff S, Townsend J, Courchesne E, Sejnowski TJ (2002) Dynamic brain sources of visual evoked responses. Science 295:690-694

Mäkinen V, Tiitinen H, May P (2005) Auditory event-related responses are generated independently of ongoing brain activity. Neuroimage 24:961-968

Massimini M, Ferrarelli F, Huber R, Esser SK, Singh H, Tononi G (2005) Breakdown of cortical effective connectivity during sleep. Science 309:2228-2232

Matthews PB (1999) The effect of firing on the excitability of a model motoneurone and its implications for cortical stimulation. J Physiol 518(Pt 3):867-882

Mazaheri A, Jensen O (2006) Posterior alpha activity is not phasereset by visual stimuli. Proc Natl Acad Sci USA 103:2948-2952

Merton PA (1951) The silent period in a muscle of the human hand. J Physiol 114:183-198

Mochizuki H, Huang YZ, Rothwell JC (2004) Interhemispheric interaction between human dorsal premotor and contralateral primary motor cortex. J Physiol 561:331-338

Nikouline V, Ruohonen J, Ilmoniemi RJ (1999) The role of the coil click in TMS assessed with simultaneous EEG. Clin Neurophysiol 110:1325-1328

Nikulin VV, Kičić D, Kähkönen S, Ilmoniemi RJ (2003) Modulation of electroencephalographic responses to transcranial magnetic stimulation: evidence for changes in cortical excitability related to movement. Eur J Neurosci 18:1206-1212

Nikulin VV, Linkenkaer-Hansen K, Nolte G, Lemm S, Müller KR, Ilmoniemi RJ, Curio G (2007) A novel mechanism for evoked responses in the human brain. Eur J Neurosci 25:3146-3154

Paus T, Jech R, Thompson CJ, Comeau R, Peters T, Evans AC (1997) Transcranial magnetic stimulation during positron emission tomography: a new method for studying connectivity of the human cerebral cortex. J Neurosci 17:3178-3184

Paus T, Sipilä PK, Strafella AP (2001) Synchronization of neuronal activity in the human primary motor cortex by transcranial magnetic stimulation: an EEG study. J Neurophysiol 86:19831990

Peinemann A, Reimer B, Loer C, Quartarone A, Munchau A, Conrad B, Siebner HR (2004) Long-lasting increase in corticospinal excitability after 1800 pulses of subthreshold $5 \mathrm{~Hz}$ repetitive TMS to the primary motor cortex. Clin Neurophysiol 115:15191526

Penfield W (1958) Some mechanisms of consciousness discovered during electrical stimulation of the brain. Proc Natl Acad Sci USA 44:51-66

Pfurtscheller G, Lopes da Silva FH (1999) Event-related EEG/MEG synchronization and desynchronization: basic principles. Clin Neurophysiol 110:1842-1857

Quartarone A, Bagnato S, Rizzo V, Morgante F, Sant'angelo A, Battaglia F, Messina C, Siebner HR, Girlanda P (2005) Distinct changes in cortical and spinal excitability following highfrequency repetitive TMS to the human motor cortex. Exp Brain Res 161:114-124

Raij T, Karhu J, Kičić D, Lioumis P, Julkunen P, Lin FH, Ahveninen $\mathrm{J}$, Ilmoniemi RJ, Mäkelä JP, Hämäläinen $\mathrm{M}$, Rosen BR, Belliveau JW (2008) Parallel input makes the brain run faster. Neuroimage 40:1792-1797

Ramos-Estebanez C, Merabet LB, Machii K, Fregni F, Thut G, Wagner TA, Romei V, Amedi A, Pascual-Leone A (2007) Visual phosphene perception modulated by subthreshold crossmodal sensory stimulation. J Neurosci 27:4178-4181
Regan D (1989) Human brain electrophysiology_evoked potentials and evoked magnetic fields in science and medicine. Elsevier, New York

Romei V, Murray MM, Merabet LB, Thut G (2007) Occipital transcranial magnetic stimulation has opposing effects on visual and auditory stimulus detection: implications for multisensory interactions. J Neurosci 27:11465-11472

Romei V, Brodbeck V, Michel C, Amedi A, Pascual-Leone A, Thut G (2008a) Spontaneous fluctuations in posterior alpha-band EEG activity reflect variability in excitability of human visual areas. Cereb Cortex 18:2010-2018

Romei V, Rihs T, Brodbeck V, Thut G (2008b) Resting electroencephalogram alpha-power over posterior sites indexes baseline visual cortex excitability. Neuroreport 19:203-208

Rosanova M, Casali A, Bellina V, Resta F, Mariotti M, Massimini M (2009) Natural frequencies of human corticothalamic circuits. J Neurosci 29:7679-7685

Rosenthal J, Waller HJ, Amassian VE (1967) An analysis of the activation of motor cortical neurons by surface stimulation. J Neurophysiol 30:844-858

Roth BJ, Pascual-Leone A, Cohen LG, Hallett M (1992) The heating of metal electrodes during rapid-rate magnetic stimulation: a possible safety hazard. Electroencephalogr Clin Neurophysiol 85:116-123

Sack AT, Kohler A, Linden DE, Goebel R, Muckli L (2006) The temporal characteristics of motion processing in hMT/V5+: combining fMRI and neuronavigated TMS. Neuroimage 29: $1326-1335$

Sack AT, Cohen Kadosh R, Schuhmann T, Moerel M, Walsh V, Goebel R (2009) Optimizing functional accuracy of TMS in cognitive studies: a comparison of methods. J Cogn Neurosci 21:207-221

Sauseng P, Klimesch W, Stadler W, Schabus M, Doppelmayr M, Hanslmayr S, Gruber WR, Birbaumer N (2005) A shift of visual spatial attention is selectively associated with human EEG alpha activity. Eur J Neurosci 22:2917-2926

Sayers BM, Beagley HA, Henshall WR (1974) The mechanism of auditory evoked EEG responses. Nature 247:481-483

Scherg M (1992) Functional imaging and localization of electromagnetic brain activity. Brain Topogr 5:103-111

Schürmann M, Nikouline VV, Soljanlahti S, Ollikainen M, Başar E, Ilmoniemi RJ (2001) EEG responses to combined somatosensory and transcranial magnetic stimulation. Clin Neurophysiol 112:19-24

Shah AS, Bressler SL, Knuth KH, Ding M, Mehta AD, Ulbert I, Schroeder CE (2004) Neural dynamics and the fundamental mechanisms of event-related brain potentials. Cereb Cortex 14:476-483

Siebner HR, Peller M, Willoch F, Minoshima S, Boecker H, Auer C, Drzezga A, Conrad B, Bartenstein P (2000) Lasting cortical activation after repetitive TMS of the motor cortex: a glucose metabolic study. Neurology 54:956-963

Silvanto J, Muggleton NG (2008) A novel approach for enhancing the functional specificity of TMS: revealing the properties of distinct neural populations within the stimulated region. Clin Neurophysiol 119:724-726

Silvanto J, Pascual-Leone A (2008) State-dependency of transcranial magnetic stimulation. Brain Topogr 21:1-10

Silvanto J, Lavie N, Walsh V (2006) Stimulation of the human frontal eye fields modulates sensitivity of extrastriate visual cortex. J Neurophysiol 96:941-945

Silvanto J, Muggleton NG, Cowey A, Walsh V (2007) Neural adaptation reveals state-dependent effects of transcranial magnetic stimulation. Eur J Neurosci 25:1874-1881

Simelius K, Ahonen A, Huotilainen M, Ilmoniemi R, Karp P, Kartano H, Katila T, Montonen J, Nenonen J, Näätänen R, Paavola M, 
Standertskjold-Nordenstam C-G (1995) BioMag: functional brain and heart research in clinical environment. In: IEEE 17th annual conference, vol 2, Engineering in Medicine and Biology Society, Montreal, Canada, pp 929-930

Sparing R, Buelte D, Meister IG, Paus T, Fink GR (2008) Transcranial magnetic stimulation and the challenge of coil placement: a comparison of conventional and stereotaxic neuronavigational strategies. Hum Brain Mapp 29:82-96

Strafella AP, Paus T, Barrett J, Dagher A (2001) Repetitive transcranial magnetic stimulation of the human prefrontal cortex induces dopamine release in the caudate nucleus. J Neurosci 21:RC157

Tam HW, Webster JG (1977) Minimizing electrode motion artifact by skin abrasion. IEEE Trans Biomed Eng 24:134-139

Tamas G, Lorincz A, Simon A, Szabadics J (2003) Identified sources and targets of slow inhibition in the neocortex. Science 299:1902-1905

Tamburin S, Manganotti P, Zanette G, Fiaschi A (2001) Cutaneomotor integration in human hand motor areas: somatotopic effect and interaction of afferents. Exp Brain Res 141:232-241

Taylor PC, Nobre AC, Rushworth MF (2007) FEF TMS affects visual cortical activity. Cereb Cortex 17:391-399

Taylor PC, Walsh V, Eimer M (2008) Combining TMS and EEG to study cognitive function and cortico-cortico interactions. Behav Brain Res 191:141-147

Thiel A, Haupt WF, Habedank B, Winhuisen L, Herholz K, Kessler J, Markowitsch HJ, Heiss WD (2005) Neuroimaging-guided rTMS of the left inferior frontal gyrus interferes with repetition priming. Neuroimage 25:815-823

Thut G, Miniussi C (2009) New insights into rhythmic brain activity from TMS-EEG studies. Trends Cogn Sci 13:182-189

Thut G, Pascual-Leone A (2009) A review of combined TMS-EEG studies to characterize lasting effects of repetitive TMS and assess their usefulness in cognitive and clinical neuroscience. Brain Topogr. doi:10.1007/s10548-009-0115-4

Thut G, Northoff G, Ives JR, Kamitani Y, Pfennig A, Kampmann F, Schomer DL, Pascual-Leone A (2003a) Effects of single-pulse transcranial magnetic stimulation (TMS) on functional brain activity: a combined event-related TMS and evoked potential study. Clin Neurophysiol 114:2071-2080

Thut G, Theoret H, Pfennig A, Ives J, Kampmann F, Northoff G, Pascual-Leone A (2003b) Differential effects of low-frequency
rTMS at the occipital pole on visual-induced alpha desynchronization and visual-evoked potentials. Neuroimage 18:334-347

Thut G, Ives JR, Kampmann F, Pastor MA, Pascual-Leone A (2005) A new device and protocol for combining TMS and online recordings of EEG and evoked potentials. J Neurosci Methods 141:207-217

Tiitinen H, Virtanen J, Ilmoniemi RJ, Kamppuri J, Ollikainen M, Ruohonen J, Näätänen R (1999) Separation of contamination caused by coil clicks from responses elicited by transcranial magnetic stimulation. Clin Neurophysiol 110:982-985

Tokimura H, Di Lazzaro V, Tokimura Y, Oliviero A, Profice P, Insola A, Mazzone P, Tonali P, Rothwell JC (2000) Short latency inhibition of human hand motor cortex by somatosensory input from the hand [published erratum appears in J Physiol (Lond) 2000 May 1;524 Pt 3:942]. J Physiol (Lond) 523:503-513

Tononi G, Cirelli C (2003) Sleep and synaptic homeostasis: a hypothesis. Brain Res Bull 62:143-150

Tononi G, Cirelli C (2006) Sleep function and synaptic homeostasis. Sleep Med Rev 10:49-62

Van Der Werf YD, Paus T (2006) The neural response to transcranial magnetic stimulation of the human motor cortex. I. Intracortical and cortico-cortical contributions. Exp Brain Res 175:231-245

Veniero D, Bortoletto M, Miniussi C (2009) TMS-EEG co-registration: on TMS-induced artifact. Clin Neurophysiol 120:13921399

Verleger R, Kuniecki M, Moller F, Fritzmannova M, Siebner HR (2009) On how the motor cortices resolve an inter-hemispheric response conflict: an event-related EEG potential-guided TMS study of the flankers task. Eur J Neurosci 30:318-326

Virtanen J, Ruohonen J, Näätänen R, Ilmoniemi RJ (1999) Instrumentation for the measurement of electric brain responses to transcranial magnetic stimulation. Med Biol Eng Comput 37:322-326

von Stein A, Chiang C, Konig P (2000) Top-down processing mediated by interareal synchronization. Proc Natl Acad Sci USA 97:14748-14753

Yeragani VK, Cashmere D, Miewald J, Tancer M, Keshavan MS (2006) Decreased coherence in higher frequency ranges (beta and gamma) between central and frontal EEG in patients with schizophrenia: a preliminary report. Psychiatry Res 141:53-60 